\title{
Batch-injection stripping voltammetry (tube-less flow-injection analysis) of trace metals with on-line sample pretreatment
}

\author{
Marek Trojanowicz $^{\mathrm{a}, \mathrm{b}, *}$, Przemysław Koźmiński ${ }^{\mathrm{a}}$, Humberto Dias ${ }^{\mathrm{c}}$, Christopher M.A. Brett $^{\mathrm{c}, \mathrm{d}}$ \\ a Department of Chemistry, Warsaw University, Pasteura 1, 02-093 Warsaw, Poland \\ ${ }^{\mathrm{b}}$ Institute of Nuclear Chemistry and Technology, Dorodna 16, 03-195 Warsaw, Poland \\ ${ }^{\mathrm{c}}$ Instituto Pedro Nunes, 3030-199 Coimbra, Portugal \\ ${ }^{\mathrm{d}}$ Department of Chemistry, University of Coimbra, 3004-535 Coimbra, Portugal
}

Available online 6 October 2005

\begin{abstract}
The most essential limitation of batch-injection analysis (BIA) methodology compared to other flow methods (CFA, FIA, SIA) is the lack of possibility of on-line sample processing in the measuring system. Some procedures of on-line sample pretreatment in BIA are possible by changing the plastic tip of the automatic micropipette used for sample injection into a flow-through reactor, e.g. by packing it with a bed of a solid sorbent. This concept is employed in the voltammetric stripping determinations of trace metals using a bed of commercial chelating resin Chelex-100. It was found that, besides the electrochemical preconcentration of analytes in the form of amalgams on the surface of mercury thin film electrodes, an approximately 10-fold additional preconcentration can be achieved on the packed sorbent bed by using different volumes of aspirated sample solution and eluent. This procedure allows also efficient elimination of some matrix effects.
\end{abstract}

(C) 2005 Elsevier B.V. All rights reserved.

Keywords: Batch-injection analysis; Stripping voltammetry; Solid-phase extraction; Trace metals

\section{Introduction}

Among the various methods of laboratory flow analysis, already under development for almost half a century, particularly great interest has focused on flow-injection methods [1]. A small volume of the sample is injected into a hydraulic system where, in the course of flow which is imposed mechanically, gravitationally or by pressure difference, a sample can be processed on-line on its way to the detector. The fundamental advantages of flow methods of analysis in such systems are: mechanically carrying out various processes with the injected sample, a high analysis efficiency, and reduction of contamination of the measuring system, especially important for trace analytes. In some cases described in the extended literature, a flow-injection analytical measurement method can improve the detection limit or even the selectivity compared to a non-flow method using the same methodology. The evidence of common interest within the analytical community in flow-injection analysis (FIA) or sequential analysis (SIA) is the thousands of original research papers in this

\footnotetext{
* Corresponding author. Tel.: +48 22822 3532; fax: +48 228223532.

E-mail address: trojan@ chem.uw.edu.pl (M. Trojanowicz).
}

field, manufactured dedicated instrumentation and the inclusion of these methods into different regulations and standardizations.

A less commonly used methodology of flow-injection analysis is batch-injection analysis (BIA), invented by Wang and Taha in 1991 [2]. Although in [2] it was described as "non-flow injection-based technique", because of the method of sample introduction, and the fact that detection is carried out during the flow of the sample segment over the detector surface, it should rather be described as tube-less flow-injection analysis. The basic feature of this measuring technique is the injection of a small volume of analyte sample by an automatic micropipette directly onto the sensing surface of the detector. The detecting device, with an active surface, is immersed in a large volume of blank solution. The detector records a transient signal in the shape of a sharp peak during the flow of sample over the detector surface. The advantage of such measurements is their short time, even in comparison to conventional flow-injection analysis, while a drawback seems to be a limited possibility of sample on-line processing. The advantage of BIA-ASV compared to FIA-ASV or SIA-ASV is that there is no need for a continuously flowing carrier solution which minimizes reagent consumption and avoids the need for pumping systems. In the pioneering work on BIA, its application with amperometric and 
potentiometric detection have been described; however, nowadays also applications with thermometric and optical detections can be found. Among various applications reported so far, the most widely described systems are with amperometric e.g. [3-6] and voltammetric [7-16] detection.

In BIA voltammetric and amperometric measurements working disk electrodes are employed in a wall-jet arrangement with injection of sample volume 10-50 $\mu$ l. In optimized conditions better dynamic characteristics can be obtained for BIA measurements than for FIA, and an additional advantage is the possibility to use electrolyte-free sample solutions [2]. The signal magnitude depends significantly on the micropipette tip-electrode distance and injection volume and injection rate [3]. The use of a working electrode with immobilized enzyme allows an on-line biocatalytic analyte derivatization in BIA measurements $[2,4]$. The use of a rotating disk electrode allows injection rates of several thousand samples per hour to be achieved [5]. BIA with cyclic voltammetry can aid in mechanistic studies, while combination of BIA with a square-wave voltammetry scan during the injection period provides a continuous voltammetric profile from a single injection [6].

In BIA anodic stripping voltammetry (BIA-ASV) measurements, during injection the accumulation of analytes on the electrode surface takes place as result of electrodeposition or of adsorption, and then a potential scan is applied in the positive direction. This is not a purely flow measurement, since only the analyte accumulation on the electrode surface is carried out in flow conditions, whereas the stripping step is carried out in quiescent solution. In the application of ASV to trace metal analysis, most often the electrochemical reduction of analyte metal ions to amalgams at mercury thin film electrode (MTFE) is employed [7-13], but the rotating disc electrode has also been used [15].

Typical BIA-ASV determinations are carried out by injection of sample from a motorized electronic micropipette onto an MTFE held at an appropriate potential for reduction of metal ions, and then employing a fast anodic square-wave voltammetric scan in the stripping step, BIA-SWASV [7]. The use of square-wave stripping minimizes the oxygen background contribution. A mercury film can be formed on a glassy carbon electrode substrate in situ in an assembled BIA detector cell [8]. Additionally, the covering of the glassy electrode substrate surface with Nafion prior to formation of the mercury film leads to reduction of electrode contamination by components of complex matrices [9,10]. For trace determination of $\mathrm{Ni}$ and $\mathrm{Co}$, the adsorption of their complexes with nioxime on MTFE was reported [14].

The important advantage of voltammetric stripping methods is the possibility of lowering the limit of detection (LOD) by lengthening analyte accumulation time on the detector electrode. In fast BIA-ASV measurement this is mainly achieved by increasing the sample volume, but it was already shown that above a certain sample volume, its further increase is not effective $[7,8]$.

The aim of this work is to examine the possibility of using a solid sorbent in the pipette tip for on-line preconcentration and the elimination of matrix effects in BIA-SWASV determinations of trace metals.

\section{Experimental}

\subsection{Instrumentation}

The design of the electrochemical cell used in this study was the same as employed earlier [3,11]. It was made of Perspex and contains about $40 \mathrm{ml}$ of background electrolyte. In the bottom of the cell a disk working electrode was mounted with the active surface facing upwards. In the screwed upper cover of the cell there are three holes-two for a platinum gauze counter electrode and a saturated calomel electrode (SCE) as reference electrode, and a third centrally drilled hole of conical shape to exactly fit a $200 \mu \mathrm{l}$ pipette tip for injection of samples directly over the center of the detector electrode at a tip-electrode distance of 2-3 mm. Glassy carbon disk electrodes of diameter 2.5 and $5.0 \mathrm{~mm}$ were used as working electrodes. Sample injection was carried out using a programmable motorized electronic micropipette model EPP Plus 100 EP-100 from Rainin (Emeryville, USA) with dispensing rate $24.5 \mu 1 \mathrm{~s}^{-1}$ in MULTIDISPENSE mode, as previously optimized [8]. A photograph of the BIA cell with the electronic micropipette used is shown in Fig. 1.

The BIA-SWASV measurements, together with the preparation of mercury films, were carried out using a computercontrolled potentiostat Autolab model PGSTAT 10, from Eco Chemie (Utrecht, Netherlands) with software GPES 4.5. Experiments were conducted in non-deaerated solutions at room temperature.

\subsection{Reagents}

Solutions were prepared from analytical grade reagent and Milli-Q deionized water (resistivity $\geq 18 \mathrm{M} \Omega \mathrm{cm}$ ). Stock solutions of $1 \mathrm{mM}$ cadmium nitrate and lead nitrate were prepared and diluted on the day, as appropriate. Triton X-100 was

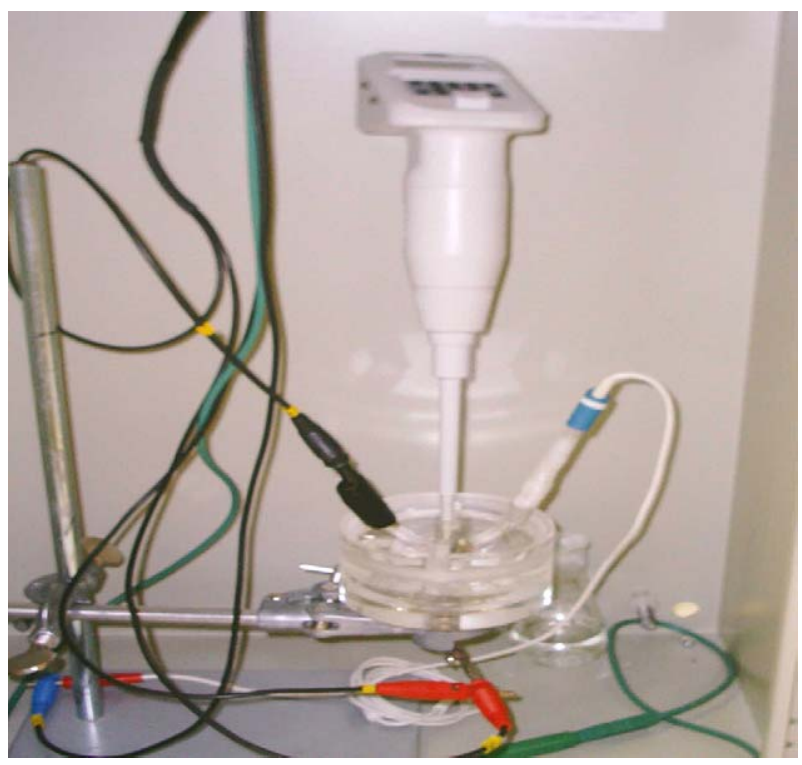

Fig. 1. The detection cell for batch-injection anodic stripping voltammetry with motorized electronic micropipette used for sample injection. 
purchased from Sigma and sodium dodecylsulphate (SDS) from Aldrich. Chelex-100, sodium form, particle size 100-200 mesh was purchased from Bio-Rad (Richmond, CA, USA).

\subsection{Procedures}

\subsubsection{Preparation of working electrode}

Prior to mounting in the BIA cell, the glassy carbon electrode was dry-polished using alumina powder with $0.1 \mu \mathrm{m}$ particles to a mirror-like finish and then rinsed with Milli-Q water. Mercury films were electrodeposited at the beginning of each day within the BIA cell. For this purpose, a volume of $10 \mu \mathrm{l}$ of a $0.1 \mathrm{M}$ $\mathrm{Hg}$ (II) solution in $0.1 \mathrm{M} \mathrm{KNO}_{3}-5 \mathrm{mM} \mathrm{HNO}_{3}$ was injected over the electrode held at an applied potential of $-1.0 \mathrm{~V}$ versus SCE during injection and for a further $60 \mathrm{~s}$ after the end of the injection period. It was shown earlier [8] that continuing applying the deposition potential after injection increased the efficiency of the electrodeposition step.

\subsubsection{Preparation of sorbent bed in pipette tips and analyte sorption step}

The outlet of conventional $200 \mu$ l pipette tips was blocked with a small amount (about a $1 \mathrm{~mm}$ layer) of glass wool, then the tip was filled with deionized water and $25 \mathrm{mg}$ of Chelex-100 beads were added. After a short time, to allow sedimentation to occur, the packed tip was ready to use.

For preconcentration, a given sample volume was aspirated through the bed into the pipette tip, left for about $30 \mathrm{~s}$, during which time the particles sedimented, and then the liquid in the tip was emptied to waste. For elution of retained metal ion analytes, a chosen volume of $2 \mathrm{M}$ nitric acid eluent was aspirated, and after $30 \mathrm{~s}$, to allow bead sedimentation to occur, the eluent with released trace metal ions was injected into the BIA cell.

\subsubsection{Square-wave anodic stripping voltammetric measurements}

In the accumulation step, the chosen volume of sample was injected into the BIA cell filled with background electrolyte at a $24.5 \mu \mathrm{s} \mathrm{s}^{-1}$ dispersion rate. The applied potential was $-1.0 \mathrm{~V}$ versus SCE during injection and for $30 \mathrm{~s}$ following injection, in order to maximize the fraction of species in the injected sample and remaining in the zone of the electrode surface, that is deposited. This was followed by anodic stripping using squarewave parameters as optimized earlier [8], namely frequency $100 \mathrm{~Hz}$, potential increment $2 \mathrm{mV}$ and square-wave amplitude $25 \mathrm{mV}$. Scanning was carried out from -1.0 to $-0.2 \mathrm{~V}$ when $\mathrm{Pb}$ and $\mathrm{Cd}$ were measured, or to $-0.5 \mathrm{~V}$ versus SCE in the determination of only $\mathrm{Cd}$.

\section{Results and discussion}

\subsection{Measurements without packed pipette tips}

The BIA-SWASV determinations of a number of metal ions were already reported and optimized in several papers [7-11]. In the pioneer paper on BIA-SWASV, $10 \mathrm{mM}$ acetate buffer $\mathrm{pH} 4.0$ was employed as background solution in the cell [7]. However, for in situ mercury film formation the optimum electrolyte was found to be potassium nitrate acidified with nitric acid [8-10]; hence, in this study the cell electrolyte was used $0.1 \mathrm{M} \mathrm{KNO}_{3}$ with $5 \mathrm{mM} \mathrm{HNO}_{3}$. As previously, it was shown here that the signal magnitude does not increase when the injected sample volume exceeds $50 \mu 1$ [8], so this sample volume was employed in further work. SWASV stripping current peak maxima were obtained at -0.60 and $-0.42 \mathrm{~V}$ versus SCE for $\mathrm{Cd}$ and $\mathrm{Pb}$, respectively. Examples for a mixture of $0.1 \mu \mathrm{M} \mathrm{Cd}$ and $\mathrm{Pb}$, and also individual responses to this analytes in the concentration range from 0.1 to $1 \mu \mathrm{M}$, are shown in Fig. 2. The equations for the calibration curves are $I=15.2 C_{\mathrm{Cd}}-4.2 \times 10^{-7} \mathrm{~A}$ and $I=28.0 C_{\mathrm{Pb}}-3.6 \times 10^{-7} \mathrm{~A}$ for $\mathrm{Cd}$ and $\mathrm{Pb}$, respectively, concentrations being expressed in mol $^{-1}$. The corresponding detection limits are 12.0 and $6.6 \mathrm{nM}$ for $\mathrm{Cd}$ and $\mathrm{Pb}$, respectively.

The signal magnitude in BIA-SWASV measurements depends on the surface area of the MTFE electrode in a nonlinear way. It was found that an increase of diameter of the working electrode from 2.5 to $5.0 \mathrm{~mm}$ results in a 7.5 -fold increase in the slope of calibration plot.

\subsection{Signal enhancement by on-line preconcentration}

Signal enhancement by solid-phase extraction with different types of sorbent is widely used in flow-injection analysis with various detection schemes (see Chapter 6.5 in [1]). It can be employed as on-line sample pretreatment using flow-through reactors loaded with appropriate sorbents, or off-line prior to sample injection, by the use of syringe cartridges or extraction discs. In the most common procedures, analyte is retained from the passage of a large volume of initial sample solution, and then eluted with a suitable eluent of much smaller volume. The offline procedure can obviously be employed in BIA, but, up until now, an on-line procedure with sorbent loaded in the pipette tip used for sample injection, has not been employed in BIA measurements. The most common chelating sorbent for trace metal preconcentration, which is Chelex-100 (see e.g. $[15,16]$ ) with iminodiacetate functional groups, was used for this purpose. Pipette tips loaded with different sorbents are also commercially available but they are employed for sample clean-up in biochemical microanalysis [17].

For effectiveness of the preconcentration step, both the sample volume used for preconcentration and the volume of eluent are of great importance. As was already seen, the ASV signal reaches a maximum with an injected sample volume (i.e. eluent volume) of about $50 \mu \mathrm{l}$ [8]. The use of large pipette tips packed with sorbent allows extra preconcentration through the manipulation and aspiration of much larger initial sample volumes, leading to larger enrichment factors. Additionally, it was found that the conical shape of the sorbent bed (which is employed here) has been reported earlier in conventional FIA measurements to increase signal magnitude compared to a uniform diameter column [18]. This was attributed to reduction of analyte dispersion during elution so that higher enrichment factors could be achieved.

In this study of preconcentration for on-line treatment of samples prior to BIA-ASV, cadmium was taken as model analyte. 

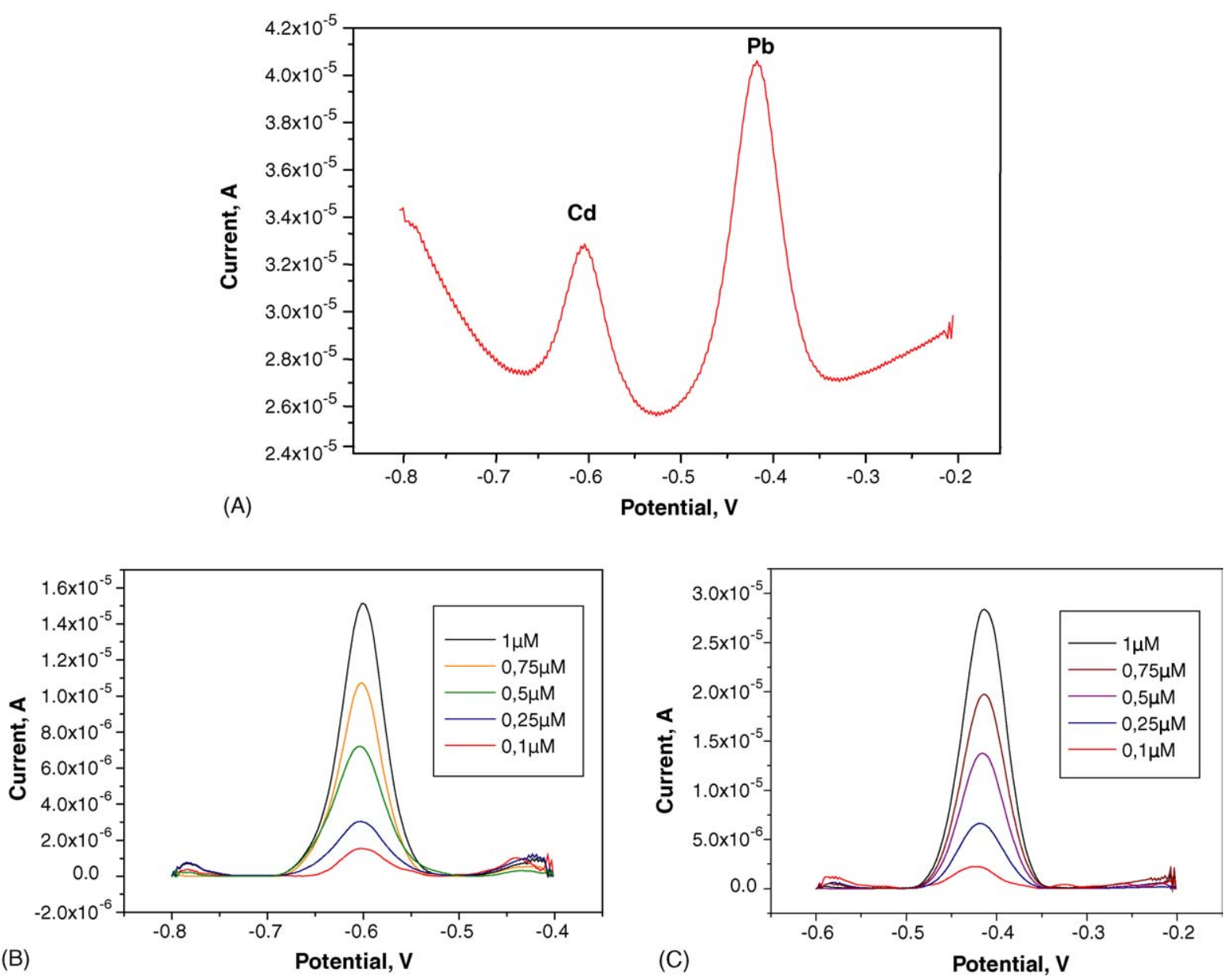

Fig. 2. BIA-SWASV recordings obtained with unloaded pipette tip for a mixture of $1.0 \mu \mathrm{M} \mathrm{Cd}$ and $\mathrm{Pb}(\mathrm{A})$, for different concentrations shown in insert of $\mathrm{Cd}(\mathrm{II})$ (B), and for different concentrations of $\mathrm{Pb}(\mathrm{II})(\mathrm{C})$. Sample injection volume $50 \mu$ l. Preconcentration $30 \mathrm{~s}$ at $-1.0 \mathrm{~V}$, other conditions as listed in Section 2.

Its sorption on a Chelex-100 bed was carried out from neutral solutions of cadmium nitrate, while for elution $2 \mathrm{M}$ nitric acid was used without further optimization since these are commonly used conditions for solid-phase extraction (SPE) of heavy metals on chelating sorbents.

In the first step, using a $0.1 \mu \mathrm{M}$ solution of $\mathrm{Cd}$ (II) and the same volume of preconcentrated sample and $2 \mathrm{M} \mathrm{HNO}_{3}$ eluent $(50 \mu \mathrm{l})$ the recovery of retained analyte was examined and in both cases, after SPE preconcentration, the peak currents obtained were about $10-15 \%$ higher than without preconcentration. This can probably be attributed to some local gradient of retained analyte in the front layer of sorbent bed, which is also reflected in the local gradient in eluted solution injected into the BIA cell. The recording of Cd signals after SPE with sorbent in the pipette tip is shown for different analyte concentrations in Fig. 3.

Since the hole in the cover of the BIA cell used in this work was fabricated with a conical shape profiled for a $200 \mu$ l pipette tip, this determined the maximum raw sample volume that could be used for SPE treatment. For aspirated sample volumes of $0.1 \mu \mathrm{M} \mathrm{Cd}(\mathrm{II})$ solutions ranging from 25 to $200 \mu \mathrm{l}$ and an eluent volume of $25 \mu \mathrm{l}$, a linear relationship between peak height and initial sample volume was observed (Fig. 4A). Based purely on the volume of injected and eluent solutions, the enrichment factor for a $200 \mu \mathrm{l}$ sample volume should be equal to 8 . Nevertheless, from comparison of signals without and with SPE preconcentration an enrichment factor equal to 10 was, in fact, obtained. The difference can be attributed, as described above, to a gradient of analyte concentration observed in solutions eluted from the bed with the front part of the sample zone more concentrated than the other.

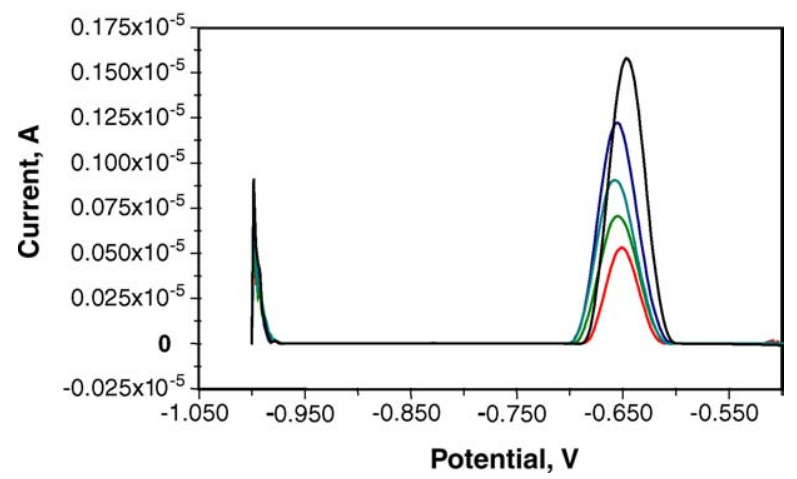

Fig. 3. BIA-SWASV voltammograms for injected Cd(II) solutions of concentration $10,25,50,75$ and $100 \mathrm{nM}$ in the system with pipette tip loaded with Chelex-100. Increasing signals correspond to increase of concentration of analyte in injected samples. Aspirated sample volumes and eluent volumes in each case $50 \mu \mathrm{l}$. Electrode diameter $5.0 \mathrm{~mm}$. 

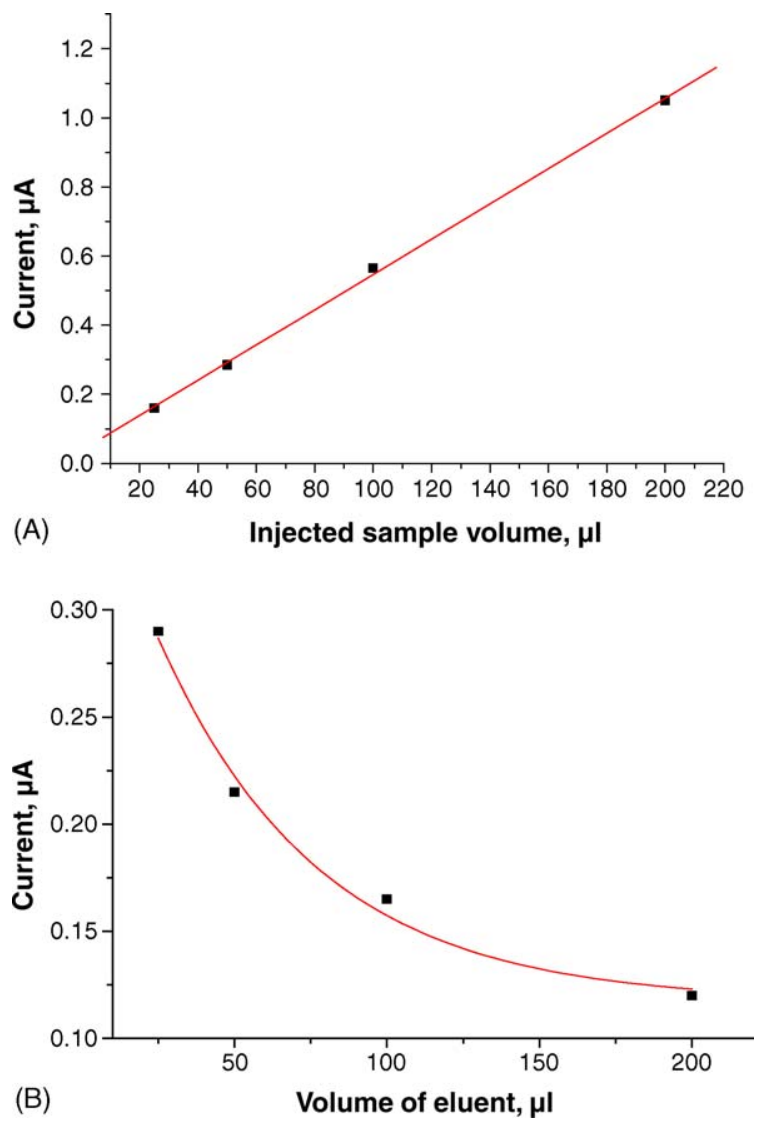

Fig. 4. Effect of sample volume and eluent volume on signal magnitude in BIASWASV measurements with sorbent loaded pipette tip and working electrode of $2.5 \mathrm{~mm}$ diameter. (A) Aspiration of $0.1 \mu \mathrm{M} \mathrm{Cd}$ (II) solutions, elution in each case with $25 \mu 1$ of $2 \mathrm{M}$ nitric acid; (B) aspiration of $200 \mu 10.1 \mathrm{mM} \mathrm{Cd(II)} \mathrm{solutions,}$ elution with $2 \mathrm{M}$ nitric acid.

The volume of eluent used should have a significant effect on signal magnitude and enrichment factor. This was examined for the preconcentration of $0.1 \mu \mathrm{M} \mathrm{Cd}(\mathrm{II})$ from $200 \mu \mathrm{l}$ initial solution, and elution volumes of $2 \mathrm{M}$ nitric acid between 10 and $100 \mu \mathrm{l}$. Theoretically, enrichment factors for 100 and $10 \mu \mathrm{l}$ of eluent should be 2 and 20, respectively, corresponding to a 10 -fold increase of stripping peak height. In practice, however, only a 2.5 -fold signal enhancement was observed (Fig. 4B). This can be explained by the overlapping effect of injected sample volume on the ASV signal in BIA measurements reported earlier, where above $50 \mu \mathrm{l}$ injected sample volume, practically no increase of signal magnitude was observed [8]. The data obtained indicate that in order to achieve the highest enrichment of analyte in BIA with on-line SPE, for a given pipette tip and depending on the possibilities of sample manipulation, the largest sample volume and the smallest eluent volume should be employed.

A similar result concerning the effect of sample volume on signal magnitude was observed for $\mathrm{Pb}(\mathrm{II})$, that gives about 10 -fold larger stripping current than $\mathrm{Cd}$ (Fig. 5A). The linearity of response was found in this case only up to about $50 \mu \mathrm{l}$ of aspirated $0.1 \mu \mathrm{M} \mathrm{Pb}(\mathrm{II})$ solution. Typical BIA-SWASV voltammograms recorded in these measurements are shown in Fig. 5B.
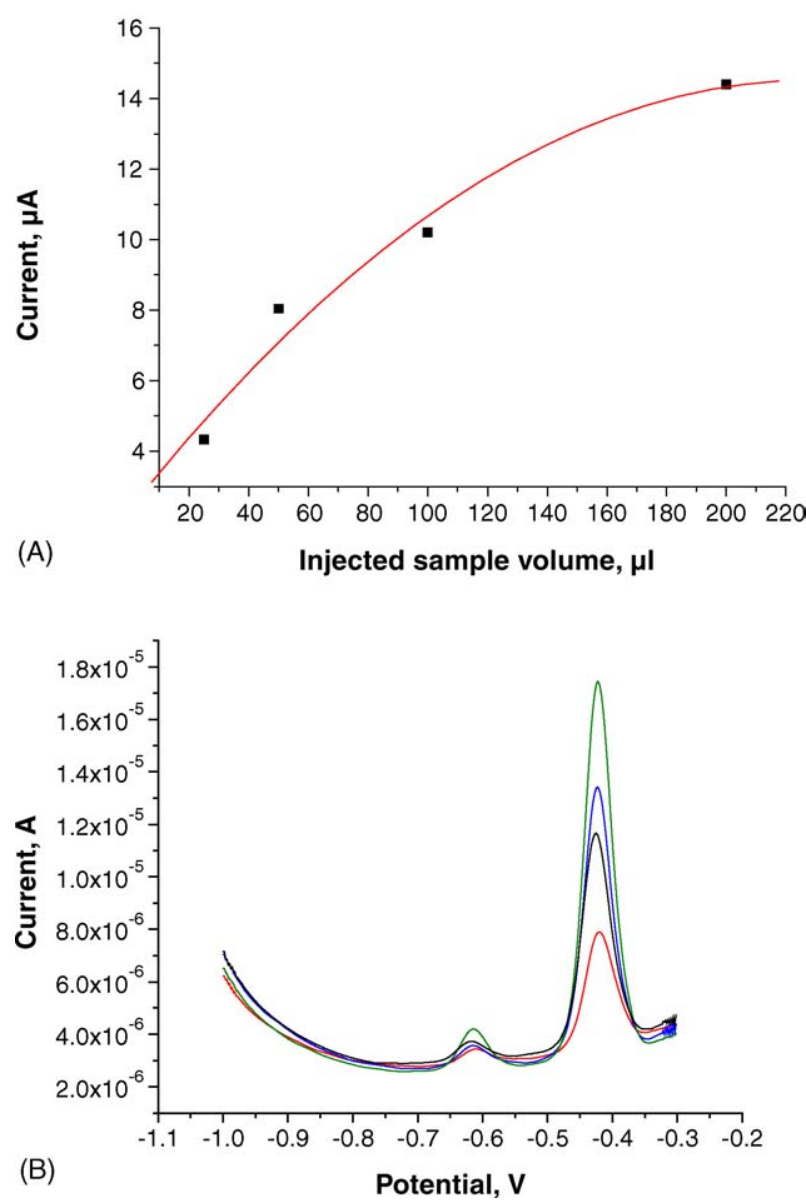

Fig. 5. (A) Effect of sample volume on signal magnitude in BIA-SWASV measurements of $\mathrm{Pb}$ with sorbent loaded pipette tip and working electrode of $2.5 \mathrm{~mm}$ diameter. Aspiration of $0.1 \mu \mathrm{M} \mathrm{Pb}$ (II) solutions, elution in each case with $25 \mu \mathrm{l}$ of $2 \mathrm{M}$ nitric acid. (B) BIA-SWASV voltammograms recorded for injection of mixtures of $0.1 \mu \mathrm{M} \mathrm{Cd}(\mathrm{II})$ and $\mathrm{Pb}(\mathrm{II})$ of volumes $25,50,100$ and $200 \mu \mathrm{l}$ and eluted with $25 \mathrm{ml} 2 \mathrm{M}$ nitric acid.

\subsection{Elimination of matrix effects with packed pipette tips}

The ASV measurements and detection limit can be significantly affected by matrix effects of other components present in the sample and the contamination of reagents. The effect of the presence of surfactants and proteins, shown for Cd(II) determination, caused a decrease of signal magnitude, which can be partly reduced using an MTFE covered with a Nafion layer [9]. Even with this type of modified MTFE, in the BIA-ASV determination of $\mathrm{Pb}$ (II) the addition of vitamin solution caused an increase of the peak currents obtained, while organic extract of marine algae decreased them [10]. It can be expected, however, that the inclusion of the SPE on-line step in BIA-ASV measurements can eliminate some of these effects.

An interesting observation was made in the initial stages of this study when BIA-ASV measurements without SPE were compared to measurements with sorbent packed tips carried out with the same $50 \mu \mathrm{l}$ volume of preconcentrated solution and nitric acid eluent. For measurements of $\mathrm{Cd}(\mathrm{II})$ content in deionized water and natural surface water, the results obtained (Fig. 6) show an effect similar to eight- to nine-fold preconcen- 


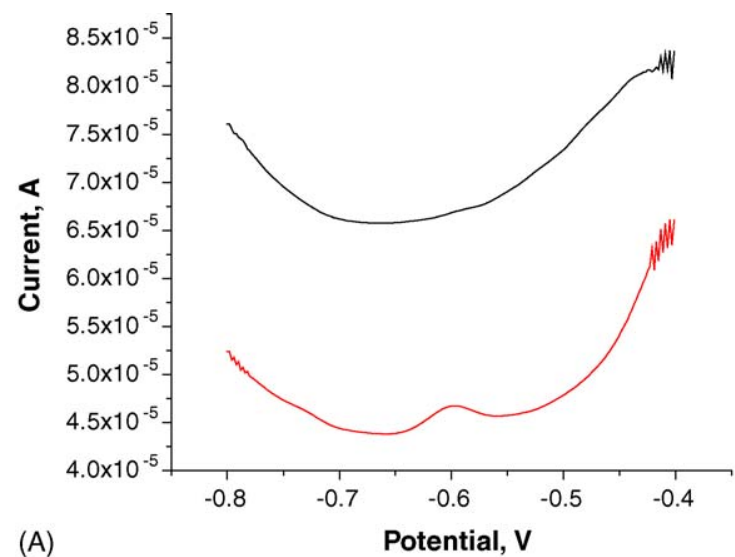

(A)

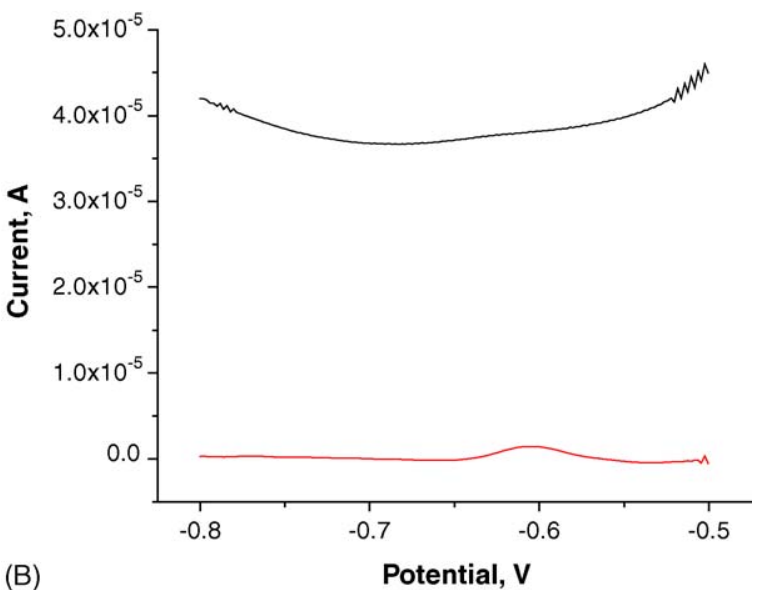

(B)

Potential, V

Fig. 6. BIA-SWASV voltammograms recorded for determination of Cd in deionized water (A) and natural surface water sample (B). Aspirated sample volume and eluent volume $50 \mu \mathrm{l}$. Upper curves obtained with non-loaded pipette tips, lower curves with the use of sorbent loaded pipette tips.

tration. The ASV recordings plotted on the same peak current scale show not only an apparent preconcentration effect (higher peaks) when sorbent packed tips were used, but also a significant decrease of background current after sorption and elution. Additional clean-up of the sample during the sorption process results in improvement of detection limit without preconcentration of analyte.

The removal of interferences from analyzed samples depends on their affinity to the sorbent employed for analyte preconcentration. Similarly to earlier studies on Nafion modified MTFEs [9], the influence of model interferents which suppress the $\mathrm{Cd}$ signal in ASV measurements was investigated-the anionic surfactant SDS and the neutral surfactant Triton X-100. Measurements were carried out for $0.1 \mu \mathrm{M} \mathrm{Cd}(\mathrm{II})$ containing different concentrations of surfactants, using the same $50 \mu$ l volume of preconcentrated sample and $2 \mathrm{M}$ nitric acid eluent. As is shown in the ASV traces in Fig. 7, the two surfactants behave differently. The well-shaped and reproducible peaks observed for $\mathrm{Cd}(\mathrm{II})$ injected with various levels of SDS suggest that negatively charged SDS is not retained on Chelex-100 for the SDS concentration range tested from 1 to $100 \mu \mathrm{M}$. However, in the presence of neutral Triton X-100 the measurements show a strong interfering effect on $\mathrm{Cd}$ determination. This can be interpreted either
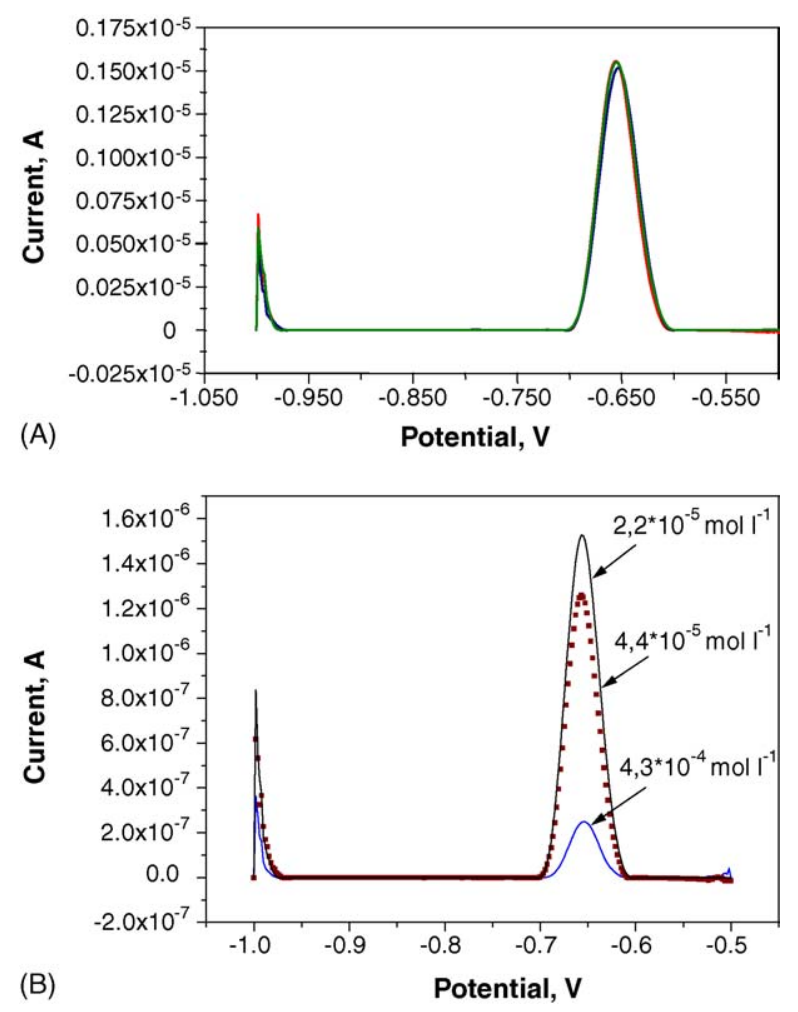

Fig. 7. Effect of the presence of surfactants in analyte samples. BIA-SWASV voltammograms recorded for $0.1 \mu \mathrm{M} \mathrm{Cd}(\mathrm{II})$ aspirated solutions in the presence of 1,10 and $100 \mu \mathrm{M}$ SDS (A), and in the presence of 22,44 and $430 \mu \mathrm{M}$ of Triton X-200 (B). Aspirated sample volume and eluent volume $50 \mu \mathrm{l}$.

as interference of non-retained surfactant with MTFE or incomplete sorption of $\mathrm{Cd}(\mathrm{II})$ in the presence of surfactant. The second of these hypotheses was confirmed by AAS measurements with electrothermal atomization of $\mathrm{Cd}$ in samples eluted from the sorbent. With an increase of Triton content in the preconcentrated sample, the amount of eluted Cd(II) decreased. Thus, the sorption of neutral surfactant on sorbent particles decreases the sorption of Cd ions, for instance, within the microchannels of the particles. This confirms the expectation that success in removal of interferences by retention on the solid sorbent depends on the affinity of the interfering species to the sorbent.

\subsection{Example applications}

Two examples of application of BIA-SWASV using pipette tips loaded with chelating sorbent will now be described for the trace determination of Cd(II). Since in both cases the same $50 \mu \mathrm{l}$ volume of sample and eluent was used, the enhancement of the ASV signal can be attributed to a decrease of background current and removal of interferences.

Fig. 8 shows the effect of the use of a sorbent-packed tip on the determination of $\mathrm{Cd}(\mathrm{II})$ in a water sample from an industrial canal obtained in measurements with the same volume $50 \mu \mathrm{l}$ of injected sample and eluent. There is an evident decrease in the background current, and possibly also the elimination of interfering effects.

Using the BIA-ASV method with sorbent-packed pipette tips, Cd(II) was also determined in a Certified Reference 


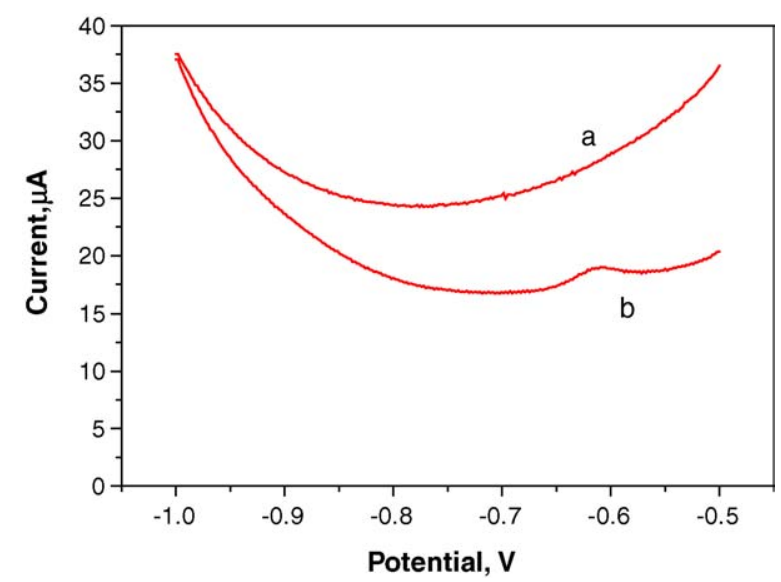

Fig. 8. BIA-SWASV voltammograms obtained for water samples from industrial canal with the use of unloaded pipette tip (a) and with sorbent loaded tip (b). Aspirated sample volume and $2 \mathrm{M}$ nitric acid eluent $50 \mu \mathrm{l}$.

Material-surface water TM-25.2 from the Natural Water Research Institute of Canada, with certified Cd(II) content $8.7 \mathrm{ppb}$. The quantitative determination was based on calibration curve. The determined concentration of $8.8 \mathrm{ppb}$ (R.S.D. $2.8 \%, n=7)$ agreed well with the certified value. In the same conditions for injection of $10 \mathrm{nM} \mathrm{Cd}(\mathrm{II})$ solution, the limit of detection (LOD) for Cd(II) was evaluated as $4.3 \mathrm{nM}(0.47 \mathrm{ppb})$ for $\mathrm{S} / \mathrm{N}=3$. As was shown above, a larger enrichment can be observed by increasing the sample volume up to $200 \mu \mathrm{l}$, that leads to an improvement in the LOD down to $0.1 \mathrm{ppb}$.

\section{Conclusions}

Although it is less commonly used than FIA or SIA, batchinjection analysis or tube-less flow-injection analysis offers a valuable improvement to anodic stripping voltammetry for trace metal determinations. The use of micropipette tips loaded with chelating sorbent, offers some additional opportunities for signal enhancement and method improvement compared to BIASWASV without sorbent. The most obvious is the lowering of detection limit by solid-phase extraction. It was also shown that the use of loaded tips allows a decrease in background cur- rent in ASV determination, resulting in a further improvement: retention of analyte on the sorbent bed can also be effective in elimination of interferences present in samples with complex matrices.

\section{Acknowledgements}

The authors acknowledge the European Union Erasmus Scheme for financing the study visit of P.K. in the University of Coimbra. The authors also thank Dr. K. Kilian, Department of Chemistry, Warsaw University for his help in ETAAS measurements.

\section{References}

[1] M. Trojanowicz, Flow Injection Analysis. Instrumentation and Applications, World Scientific Publishing, Singapore, 2000.

[2] J. Wang, Z. Taha, Anal. Chem. 63 (1991) 1053.

[3] C.M.A. Brett, A.M. Oliveira Brett, L.C. Mitoseriu, Electroanalysis 7 (1995) 225.

[4] A. Amine, J.-M. Kauffmann, G. Palleschi, Anal. Chim. Acta 273 (1993) 213.

[5] L. Chen, J. Wang, L. Angnes, Electroanalysis 3 (1991) 773.

[6] C.M.A. Brett, A.M. Oliveira Brett, L.C. Mitoseriu, Anal. Chem. 66 (1994) 3145.

[7] J. Wang, J. Lu, L. Chen, Anal. Chim. Acta 259 (1992) 123.

[8] C.M.A. Brett, A.M. Oliveira Brett, L. Tugulea, Anal. Chim. Acta 322 (1996) 151.

[9] C.M.A. Brett, A.M. Oliveira Brett, F.M. Matysik, S. Matysik, S. Kumbhat, Talanta 43 (1996) 2015.

[10] C.M.A. Brett, D.A. Fungaro, J.M. Morgado, M.H. Gil, J. Electroanal. Chem. 468 (1999) 26.

[11] C.M.A. Brett, J.M. Morgado, J. Appl. Toxicol. 20 (2000) 477.

[12] R.M. Barbosa, L.M. Rosario, C.M.A. Brett, A.M. Oliveira Brett, Analyst 121 (1996) 1789.

[13] C.M.A. Brett, A.M. Oliveira Brett, L. Tugulea, Electroanalysis 8 (1996) 639.

[14] Y.I. Tyryan, E.M. Strochkova, I. Kuselman, A. Shednhar, Fresen. J. Anal. Chem. 354 (1996) 410.

[15] C. Gueguen, J. Dominik, D. Perret, Fresen. J. Anal. Chem. 370 (2001) 909.

[16] M. Soylak, Anal. Lett. 37 (2004) 1203.

[17] Varian Inc., OMIX Pipette tips for micro extractions, http://www. varianinc.com.

[18] Z. Fang, B. Weltz, J. Anal. Atom. Spectrom. 4 (1989) 543. 